\title{
L'éthique dans le système éducatif espagnol
}

\section{Luis Maria Cifuentes Perez}

Traducteur : Claudine Adam

\section{OpenEdition}

\section{Journals}

Édition électronique

URL : http://journals.openedition.org/ries/4168

DOI : 10.4000/ries.4168

ISSN : 2261-4265

Éditeur

Centre international d'études pédagogiques

Édition imprimée

Date de publication : 5 mars 1995

Pagination : 111-117

ISSN : 1254-4590

Référence électronique

Luis Maria Cifuentes Perez, "L'éthique dans le système éducatif espagnol », Revue internationale d'éducation de Sèvres [En ligne], 05 | 1995, mis en ligne le 16 mars 2015, consulté le 03 mai 2019. URL : http://journals.openedition.org/ries/4168; DOI : 10.4000/ries.4168

Ce document a été généré automatiquement le 3 mai 2019.

(c) Tous droits réservés 


\title{
L'éthique dans le système éducatif espagnol
}

\author{
Luis Maria Cifuentes Perez
}

Traduction : Claudine Adam

1 La période politique et éducative que vit actuellement l'Espagne nous conduit à resituer la question des relations entre éthique, école et société dans une perspective philosophique propre à établir une cohérence entre tous ces éléments, à partir de l'analyse de la réforme du système éducatif espagnol. Mais pour ce faire, il nous faut d'abord commencer par une brève analyse historique du système éducatif antérieur à la démocratie espagnole, c'est-àdire du système éducatif du «national-catholicisme», pour ce qui touche à l'éthique. Dans notre pays, on a appelé le passage politique de la dictature à la démocratie «la transition politique »; on désigne par là la période qui va de 1975 à 1981, durant laquelle tous les citoyens espagnols ont participé d'une façon ou d'une autre à la consolidation d'un nouveau régime politique: la démocratie parlementaire et la monarchie constitutionnelle.

2 Cependant, le changement social, économique et culturel avait déjà eu lieu avant la mort du général Franco, et dès le milieu des années soixante, la jeunesse, les intellectuels, les travailleurs et de nombreux membres de l'Église catholique avaient évolué vers des formes de vie et de culture proches de la démocratie politique. On peut dire à juste titre que le changement politique espagnol a été postérieur au changement social et culturel.

\section{Le monopole moral de la religion catholique 1939-1975}

3 Pour bien comprendre la signification actuelle de l'éthique dans le système social, politique et éducatif espagnol, il faut effectuer un bref excursus historique vers le passé le plus immédiat, avant l'instauration de la démocratie en Espagne; c'est-à-dire analyser 
brièvement le rôle de l'Église et de la religion catholique et son influence dans la vie politique, sociale et éducative pendant la dictature franquiste (1939-1975).

C'est l'Église catholique qui, durant les quarante années de la dictature espagnole, a eu le monopole du système des valeurs et des règles morales imposées à toute la population par tous les moyens. N'oublions pas que le système éducatif espagnol apparu après la guerre civile fut élaboré et mis en place presqu'exclusivement par l'Église catholique qui forma dans ses collèges les enfants des classes moyennes des villes de tout le pays jusqu'à la fin des années soixante. Tout au long de l'enseignement primaire et secondaire, l'ensemble de la population scolaire des années quarante, cinquante et soixante fut soumise à un endoctrinement systématique. C'est pourquoi on a pu dire avec raison que c'est à partir du système éducatif franquiste que l'idéologie du " national-catholicisme » s'est étendue à tout le tissu social espagnol.

5 Pourtant, à partir du concile Vatican II (1963-1965), sous l'impulsion du catholicisme progressiste qui préconisait l'adaptation au monde, un certain changement moral, un certain renouvellement du mode de vie catholique était apparu. En fait, à la mort du général Franco en 1975, une transformation assez significative des mœurs, des règles et des valeurs morales s'était déjà produite dans la jeunesse. Même dans la vie politique, l'élan démocratique et la critique de la dictature venaient en grande partie des secteurs progressistes catholiques très liés aux organisations catholiques opposées à la dictature politique. Au cours des années soixante et du début des années soixante-dix, apparurent des grèves, des manifestations et des réunions clandestines sous l'égide de secteurs de l'Église catholique, qui contribuèrent grandement à affaiblir le régime autoritaire du franquisme. Durant les quinze dernières années du franquisme (1960-1975), le combat pour les libertés regroupa catholiques, communistes, socialistes et d'autres démocrates espagnols qui désiraient un changement politique et éthique dans la société espagnole.

Dans le contexte politique et éducatif de cette période, l'éthique n'eut jamais le statut académique d'une discipline philosophique et autonome. La philosophie morale transmise dans tous les cours de religion et morale catholiques était présentée comme la seule vérité morale sur les mœurs et la conduite de toute la population espagnole. Et l'on confia aussi le théâtre, le cinéma, la télévision, la presse, les revues et tous les secteurs culturels extérieurs au domaine scolaire à des censeurs ecclésiastiques qui veillaient à l'orthodoxie catholique de ces formes d'expression culturelle.

7 La religion catholique fournit au système éducatif espagnol les moyens idéologiques de légitimation de la dictature jusqu'au milieu de la décennie des années soixante. Toute réflexion éthico-philosophique qui aurait pu mettre en doute la vérité morale du catholicisme était interdite et censurée dans le système éducatif. Les manuels scolaires de 1940 à 1970 sont le meilleur exemple de dogmatisme antiphilosophique et d'intolérance à l'égard des autres idées. Quelques-uns des meilleurs écrivains et autres intellectuels espagnols tels que Miguel de Unamuno ou Benito Pérez Galdos furent considérés par l'école «nationale-catholique » comme des "hérétiques immoraux » qui ne méritaient pas d'être étudiés par les élèves espagnols.

8 Quant aux rapports entre morale et politique dans le contexte du système éducatif " national-catholique ", on peut dire qu'elles formaient un bloc homogène et unitaire. Les théories politiques et sociales enseignées dans les classes à tous les élèves reposaient sur la doctrine catholique de l'Église et le concept de démocratie libérale était méprisé par 
tous les théoriciens du régime franquiste, étant donné que les principes du Mouvement national étaient la seule expression de la vérité politique.

\section{Éthique et société espagnole aujourd'hui}

9 Les changements politiques, sociaux et économiques que la société espagnole a connus, de 1975 à nos jours, ont posé une série de problèmes nouveaux qui, dans le cas espagnol, sont dignes d'intérêt.

\section{La perte de la morale catholique}

10 Ce fait constitue, à mon avis, un des phénomènes actuels les plus palpables de la vie sociale et culturelle espagnoles. L'influence d'une religion qui offrait des valeurs morales absolues, vraies et uniques a presque totalement disparu de cette vie sociale et culturelle espagnole. La preuve en est que la censure exercée par la religion et la morale catholiques pendant le franquisme a été complètement abolie dans toutes les manifestations culturelles (livres, journaux, revues, cinéma et télévision). En vingt ans, la transformation des mœurs et des critères moraux de la société espagnole a été vertigineuse. La loi sur le divorce et la loi sur l'avortement sont ici exemplaires. Ces deux lois approuvées par le Parlement espagnol sont rejetées par la doctrine religieuse et morale de l'Église catholique, mais l'Église catholique a dû les accepter car le Tribunal constitutionnel les a déclarées conformes à la Constitution espagnole. Le pouvoir exécutif et législatif espagnol a su garder son indépendance par rapport aux pressions que l'Église tentait d'exercer à travers les partis politiques proches de son idéologie. Pourtant, plusieurs années après l'approbation de ces lois, l'Église catholique conserve l'espoir d'un changement politique qui puisse favoriser ses thèses théologiques et ses intérêts moraux. Dans ce but, elle compte sur l'appui idéologique que lui offre un certain parti politique, même s'il n'existe en Espagne aucun groupe politique semblable à la démocratie-chrétienne italienne ou allemande. La création d'un parti aux sigles confessionnels lié au catholicisme est, pour l'instant, impossible en Espagne, l'expérience du «national-catholicisme» de l'étape franquiste étant trop récente et ayant laissé de mauvais souvenirs.

11 À l'heure actuelle, la religion catholique n'est plus obligatoire pour tous les élèves ; mais dans toutes les classes de l'educación general básica (de 6 à 14 ans) et du Bachillerato (de 14 à 17 ans) il existe une option de deux heures hebdomadaires: éthique ou religion catholique. Dans le système éducatif espagnol, la réglementation concrète de la religion et morale catholique découle des Accords entre le Saint-Siège et l'État espagnol sur les questions culturelles, traité international datant de 1978. Cependant, le status de la religion catholique et des autres confessions religieuses (évangélique, islamique et juive) est actuellement en cours de révision, compte tenu de la nouvelle loi sur l'enseignement et des accords de l'État espagnol avec les autres confessions religieuses non catholiques.

\section{Pluralisme moral et amoralité dans l'Espagne d'aujourd'hui}

La société espagnole actuelle est, sous cet aspect, semblable aux autres sociétés européennes occidentales. La tradition catholique qui a fortement imprégné toute la vie espagnole pendant plusieurs siècles semble avoir perdu toute influence sur la vie et les habitudes des citoyens et on assiste à un phénomène nouveau et étrange : l'acceptation 
du pluralisme moral actuel et en même temps un certain degré d'amoralité, de manque de critères moraux d'une grande partie de la population.

Espagnols est définitivement aboli et que l'influence dominatrice de l'Église catholique sur les jugements éthiques des citoyens de notre pays a, en grande partie, disparu surtout dans les générations espagnoles les plus jeunes. Le pluralisme moral, bien qu'il n'ait ni tradition ni fondement historique sur lequel s'asseoir, commence à s'enraciner lentement dans la société espagnole, car la démocratie politique est la voie qui permet un réel dialogue éthique entre des positions philosophiques différentes. Les Espagnols, surtout les plus jeunes, apprennent à cohabiter avec des personnes et des mentalités de culture, de langue et de religion différentes et à comprendre la signification de la démocratie : elle est le système qui autorise le dialogue entre des points de vue divergents et opposés. Le pluralisme moral est donc le produit du pluralisme politique et culturel défendu et légitimé par la Constitution espagnole de 1978. Les lois, les médias et le système éducatif lui-même sont le reflet de la pluralité des options idéologiques et philosophiques présentes dans la vie politique et sociale de l'Espagne actuelle. En outre, l'immigration croissante de Maghrébins, d'Africains et d'Hispano-américains en Espagne, pose de nouveaux problèmes de cohabitation interculturelle qui exigent l'acceptation du pluralisme culturel et moral. Peu à peu, quoique de manière encore confuse, les Espagnols prennent conscience qu'ils doivent apprendre à respecter des façons de vivre différentes de la leur et à tolérer d'autres critères éthiques de conduite morale.

En tant qu'éducateur et que professeur de philosophie, je veux pourtant souligner un problème très grave qui touche la société espagnole actuelle : l'amoralité provenant du manque de réflexion éthique. Malgré les grandes campagnes des journaux et de tous les médias qui ne cessent de parler d'éthique, de moralité et d'immoralité dans la vie publique espagnole, la majorité de la population espagnole n'a ni éducation morale ni réflexion éthique sur la vie morale individuelle ou sur les vertus nécessaires à la vie publique; la conscience critique qui aiderait à la formation du jugement moral des citoyens espagnols n'est pas développée. Ce qui nous amène aussitôt à nous demander quel est dans ce domaine, le rôle de l'école, de l'activité éducative. Collabore-t-elle ou non à la formation du jugement moral et critique des jeunes élèves ?

\section{L'éthique dans la réforme éducative actuelle}

16 Avec l'arrivée au gouvernement du parti socialiste espagnol (PSOE) en 1982, une réforme éducative de tout le système d'enseignement, de la maternelle à l'université, a été mise en route. Sous la devise de la modernisation économique et de l'adaptation à l'espace européen, l'Espagne connaît depuis lors un processus de changement éducatif qui n'a cependant pas encore été généralisé à tout le territoire espagnol. Pour comprendre la nécessité et la complexité de cette réforme éducative, il suffit de dire qu'aujourd'hui l'État espagnol est du point de vue constitutionnel un État pluriculturel, plurilingue et plurinational. 
17 Le premier changement introduit par la nouvelle loi organique générale du système éducatif (LOGSE) est l'acceptation de la pluralité culturelle et éducative espagnole avec ses langues et traditions divers (castillan, basque, catalan et galicien). C'est la première fois dans l'histoire de l'Espagne que le système éducatif n'est pas centraliste, qu'il est autonomiste, pluriel, tout en sauvegardant une certaine unité fondamentale de base.

La présence de l'éthique dans le nouveau système éducatif espagnol comporte deux aspects distincts, selon le type d'enseignement et d'élèves de l'étape éducative concernée.

Tout d'abord, l'éducation aux valeurs que souhaite offrir le nouveau système éducatif implique que les attitudes, les valeurs et les règles morales de chaque établissement scolaire fassent partie d'un Projet éducatif la réalisation duquel l'ensemble des enseignants participeraient. Dans les établissements qui appliquent déjà de façon anticipée la réforme éducative pour les jeunes de 3 à 16 ans (étapes préscolaire, primaire et secondaire obligatoire), on essaie d'appliquer ce concept d'éducation morale sous le nom de disciplines transversales, car on estime qu'elles traversent et imprègnent tout le projet curriculaire et éducatif de l'établissement. Les responsables politiques du ministère de l'éducation espagnol souhaitent que, grâce à la mise en œuvre de ce projet, tous les élèves connaissent et vivent les valeurs suprêmes implicites dans la Constitution espagnole la solidarité, l'égalité, la tolérance, etc. On cherche à ce que tous les professeurs soient réellement et effectivement conscients de la projection éthique de leur tâche éducative. En plus de ces valeurs morales, on souhaite aussi que l'école apprenne un autre type de valeurs (les valeurs esthétiques, par exemple).

Le second aspect de la formation éthique consiste en une initiation à la réflexion éthique et la vie morale. Après de nombreuses heures de discussion et de pression de la part des professeurs de philosophie, notre ministère de l'éducation a accepté que tous les élèves de dernière année de l'enseignement secondaire obligatoire (16 ans) consacrent deux heures hebdomadaires à la vie morale et la réflexion éthique. Le programme élaboré pour ce bloc thématique englobe des thèmes tels que : la genèse des valeurs morales; les systèmes et théories éthiques; l'autonomie et l'hétéronomie morales; les problèmes moraux de notre temps et les projets éthiques contemporains; l'autorité et sa légitimation; la désobéissance civile; la religion en tant que fait individuel et social.

21 Les thèmes proposés dans ce programme d'éthique philosophique ont pour but d'offrir aux élèves une approche philosophique plus dense et plus profonde des thèmes étudiés au cours des étapes éducatives antérieures (de 6 à 16 ans) et de compléter les thèmes appelés transversaux (éducation au développement, éducation à la santé, éducation sexuelle, etc.). Cependant cette discipline rencontre des problèmes : un horaire faible, deux heures par semaine; le manque d'habitude de pensée philosophique et de techniques de travail intellectuel adaptées à la philosophie chez les élèves qui sont confrontés pour la première fois cette année à la réflexion éthico-philosophique.

Avec la réforme éducative en cours, le parti socialiste a voulu transformer tous les professeurs en éducateurs moraux capables d'exercer une influence sur la formation du jugement moral et critique de tous les écoliers du pays. En principe, son idée de l'éducation est correcte, puisqu'un professeur n'est pas uniquement un spécialiste en disciplines épistémologiques, en connaissances; c'est aussi quelqu'un qui transmet, consciemment ou non, à ses élèves des attitudes, des valeurs et des règles morales. Tout enseignement et toute éducation présupposent, de façon plus ou moins explicite, une philosophie de l'action éducative. 

religieuses individuelles et des partis politiques espagnols; elle doit être avant tout civique, fruit d'un nouveau pacte social et politique qui fera émerger des valeurs communes à tous les Espagnols. Elle doit donc être le résultat d'un dialogue institutionnel entre les représentants de la politique, de la société et de la culture. La lutte contre toutes les formes de corruption morale, contre l'intolérance, contre le fanatisme, le racisme et la 
xénophobie de la nouvelle Europe réclame, de façon urgente, une nouvelle éthique humaniste, civique et universelle basée sur le respect et la dignité de la personne.

\section{RÉSUMÉS}

L'histoire récente de l'Espagne a permis d'assister à des mutations spectaculaires simultanées du régime politique, de la société et de l'école. Le statut de l'exigence éthique s'en est trouvé luiaussi bouleversé. Après la rupture avec le franquisme et la restauration de la démocratie, on a vu apparaître, sur fond d'affaiblissement de la morale catholique, un véritable pluralisme moral, qui assigne à l'école un rôle essentiel : il s'agit d'aider les élèves à fonder en raison leurs choix, dans le respect de la diversité culturelle espagnole. Une nouvelle culture éthique doit se constituer hors de toute emprise politique ou religieuse.

\section{INDEX}

Index géographique : Espagne, Europe

Mots-clés : éducation morale, éthique, histoire, pouvoir politique, système éducatif, valeur, éducation

\section{AUTEURS}

\section{LUIS MARIA CIFUENTES PEREZ}

Président de la Société espagnole des professeurs de philosophie, Madrid, Espagne 